\title{
DOR CRÔNICA E DEPRESSÃO: ESTUDO EM 92 DOENTES
}

\section{CHRONICA PAIN AND DEPRESSION: STUDY WITH 92 PATIENTS}

\author{
Cibele Andrucioli de Mattos Pimenta* \\ Maria Sumie Koizumi** \\ Manoel Jacobsen Teixeira***
}

PIMENTA, C. A. M. et al. Dor crônica e depressão: estudo em 92 doentes. Rev.Esc.Enf.USP, v. 34, n. 1, p. 76-83, mar. 2000.

\section{RESUMO}

O estudo objetivou analisar a existência de relações entre dor e depressão em noventa e dois doentes com doença oncológica avançada. Os doentes foram divididos em 2 grupos, com ou sem dor na semana anterior à entrevista. Havia dor em 62,0\% dos avaliados, a duração média do quadro álgico foi 10 meses e a maioria referiu dor moderada. Os individuos do grupo com dor apresentaram escores de depressão significativamente mais altos que os do grupo sem dor $(p<0,05)$. Doentes com mais altos escores de depressão experienciaram dor de maior intensidade $(p<00,5)$. Sintomas depressivos associaram-se e agravaram a experiência dolorosa.

PALAVRAS-CHAVE: Dor. Depressão. Avaliação da dor. Câncer.

\section{ABSTRACT}

Was examined the influence of depression in the ocurrence and pain intensity. Patients presenting advanced cancer $(n=92)$ were evaluated. The patients were divided in 2 groups, with or without pain during the week preceding the interview. Pain and depression were evaluated. Pain was observed in 62,0\% of the cases and lasted 10 months as an average. It was moderate in the majority of patients and severe in 115 of them. Depression was related with the presence and intensity of pain. Patients with pain presented higher depression scores than patients without pain $(p<0,05)$. Higher pain scores were also correlated with higher depressive scores $(p<0,05)$.

KEYWORDS: Pain. Depression. Pain measurement. Câncer

\section{INTRODUÇÃO}

Os conceitos sobre dor sofreram profundas modificações em torno das décadas de 50 e 60. Incluiram-se, no universo de facetas que configuram a sensação dolorosa, além dos aspectos físicos, os culturais e emocionais. A Sociedade Internacional para Estudo da Dor, em 1979, conceituou dor como "uma experiência sensorial e emocional desagradável que é descrita em termos de lesões teciduais, reais ou potenciais. A dor é sempre subjetiva e cada indivíduo aprende a utilizar este termo através de suas experiências traumáticas" 33 .
A dor relacionada ao câncer acomete entre $50 \%$ a $70 \%$ dos doentes em todos os estágios da doença e em torno de $70 \%$ a $90 \%$ daqueles com doença oncológica avançada ${ }^{8} \mathrm{O}$ fenômeno doloroso resulta da interpretação do aspecto físico-químico do estímulo nocivo e da interação deste com características individuais como humor, significado simbólico atribuído ao fenômeno sensitivo e aspectos culturais e afetivos dos indivíduos ${ }^{33}$

A Organização Mundial de Saúde propôs método para o alívio da dor do câncer25. Diversos estudos comprovam a eficácia do programa proposto $21,26,34,35$

\footnotetext{
*Enfermeira. Professora Associada do Departamento de Enfermagem Médico-Cirúrgica da Escola de Enfermagem da USP. Coordenadora de Enfermagem da Liga de Dor do HC-FMUSP. E-mail: parpca@usp.br

*Enfermeira. Professora Titular do Departamento de Enfermagem Médico-Cirúrgica da Escola de Enfermagem da Universidade de São Paulo.

* Neurocirurgião. Professor Doutor do Departamento de Neurologia da Faculdade de Medicina da Universidade de São Paulo.

Chefe do Ambulatório de Dor da Clínica Neurológica do Hospital das Clínicas da Faculdade de Medicina da Universidade de São Paulo.
} 
e outros relatam a inadequação do controle da dor em países desenvolvidos ${ }^{15,21}$ e subdesenvolvidos ${ }^{32}$.

As causas do insuficiente alivio da dor advêm de falhas na formação dos profissionais de saúde na área de analgesia; da apreciação inadequada ou negligência para com o problema da dor, do incorreto uso das terapias analgésicas e de falhas no modelo teórico da dor oncológica e de seu tratamento. Há, provavelmente, aspectos, além dos biológicos, como os culturais e afetivos, envolvidos na vivência e expressão da dor que, talvez, sejam negligenciados 8,14 .

As emoções mais comumente associadas à dor crônica são depressão e ansiedade ${ }^{8,10,36}$ Depressão é o diagnóstico psiquiátrico mais comum em doentes com afecções clínico-cirúrgicas ${ }^{8}, 11,30 \mathrm{E}$ freqüentemente não identificada e não tratada ${ }^{27}$. E um quadro prevalente em $4 \%$ a $5 \%$ da população geral ${ }^{30}$. Nos doentes com dor crônica a prevalência da depressão varia entre $22 \%$ e $78 \%$ segundo alguns autores $810,36 \mathrm{e}$, de acordo com outros ${ }^{24}$, entre $10 \%$ e $30 \%$. Queixas dolorosas persistentes ocorrem entre $30 \%$ e $100 \%$ dos doentes deprimidos. Sintomas depressivos intensos afetam $25 \%$ de todos os doentes com câncer e cerca de $70 \%$ daqueles com doença avançada 8,10,17,36 Imagina-se que sintomas depressivos, quando não reconhecidos e abordados adequadamente, podem dificultar seriamente o tratamento e levar os doentes a aderirem menos à terapêutica e à obtenção de resultados menos satisfatórios no controle do quadro álgico. Esta pesquisa visa à busca de possiveis associações entre a ocorrência e expressão da dor e aspectos depressivos.

\section{OBJETIVOS}

1. Avaliar, em doentes com doença oncológica avançada, a existência de associações entre a ocorrência de dor e as variáveis: sexo, idade, escolaridade, renda per capita, atividade remunerada, ter ciência do diagnóstico da neoplasia, localização do tumor primário, localização das metástases, tipo de atividade da doença e estado depressivo.

2. Verificar a existência de correlações entre a intensidade da dor e estado depressivo.

\section{METODOLOGIA}

O estudo foi desenvolvido no Ambulatório do Serviço de Radioterapia do Hospital das Clínicas da Faculdade de Medicina da Universidade de São Paulo. Foram estudados 92 doentes, em tratamento no período entre outubro de 1993 a fevereiro de
1994. Os critérios de inclusão dos doentes foram ter: doença oncológica avançada, idade mínima de 16 anos, condições físicas, capacidade de compreensão e verbalização adequadas para participar da entrevista. Considerou-se doença oncológica avançada aquela com grande extensão loco-regional ou com metástases e não apenas o doente terminal. Por doente terminal entendeu-se "aquele que é incapaz de manter suas atividades físicas ou de manter vida de relação e cuja morte é esperada a curto prazo". O consentimento do doente foi obtido verbalmente, prática usual na época em que os dados foram coletados.

A presença ou ausência de dor, na semana anterior à entrevista, foi o fator de divisão da amostra em 2 grupos:

grupo 1 - doentes que não sentiram dor na semana anterior à entrevista

grupo 2 - doentes que sentiram dor na semana anterior à entrevista

Os dados foram coletados em entrevista individual, única, realizada em ambiente privativo e previamente agendada. Os doentes foram avaliados por meio de instrumentos padronizados. O Impresso para encaminhamento permitiu caracterizar a amostra quanto à localização do tumor primário, a existência e localização de doença metastática, de doença avançada loco-regionalmente ou ambas e se a dor poderia ser atribuída ao tumor ou às metástases. O Instrumento I permitiu caracterizar a população quanto aos dados de identificação (nome, idade, sexo, endereço, renda familiar e per capita, escolaridade e atividade ocupacional), quanto ao fato de. o doente ter ou não ciência sobre o diagnóstico da neoplasia, ocorrência ou não de dor na última semana e duração do quadro álgico. Permitiu, ainda, caracterizar a intensidade da dor por meio de escala numérica graduada de 0 a 1022 $(0=$ ausência de dor e $10=$ pior dor imaginável $)$. $O$ Instrumento II é o Inventário de Depressão de BECK ${ }^{4}$. E um instrumento de auto-avaliação de estado depressivo, validado e testado em inúmeros trabalhos ${ }^{4,13,18}$ Neste estudo, foi utilizada a tradução em língua portuguesa que consta do livro "Terapia Cognitiva da Depressão" 3. O inventário é composto por 21 ítens, com 4 graus de intensidade $(0$ a 3$)$. As afirmações são graduadas para refletir a gravidade do sintoma, de neutro até intensidade máxima. O mais alto escore possivel é 63 e significa intensidade máxima da depressão. O inventário de depressão de Beck é um instrumento de auto-avaliação de estado depressivo e permite discriminar doentes deprimidos dos não deprimidos 10,13 entre $82 \%$ a $88 \%$ das vezes 13,36 . 0 ponto de corte para doentes brasileiros não foi estabelecido.

A intensidade da dor foi avaliada por meio de escala numérica de 0 a $10^{22}$ e classificada em leve (valor de 1 a 3), moderada (4 a 7) e intensa (8 a 10). 
A existência de estado depressivo foi avaliada através do inventário de BECK ${ }^{4}$. Adotaram-se valores superiores a 13 e iguais ou superiores a 21 como pontos de corte, como recomendado por BECK e outros autores 13,18

Foram aplicados diversos testes estatísticos s, 29 (Prova do Qui-quadrado - $\mathrm{x}^{2}$; Prova de MannWhitney, Prova de Kruskal-Wallis, Coeficiente de correlação por postos de Kendall Tau e Teste de Fisher). Todas as provas foram realizadas admitindo-se probabilidade de erro de primeira espécie (erro tipo I) de $5 \%$. Foram seguidas as recomendações de SIEGEL 29 quanto à adequação e aproximações possiveis em cada teste. O tratamento estatístico dos dados teve como objetivo básico a comparação de dados intergrupos (grupos 1 e 2) e intragrupo (grupo 2). As comparações intergrupos visaram a avaliar se as variáveis sócio-demográficas, da doença e afetivas associaram-se à ocorrência de dor. As comparações intragrupo visaram a identificar possiveis correlações entre a intensidade da dor e estado depressivo.

\section{RESULTADOS}

\section{Caracteristicas pessoais e da neoplasia dos doentes dos grupos 1 e 2}

Havia queixa álgica em $62,0 \%$ dos doentes. A maior parte $(66,7 \%)$ apresentou dor moderada (4 a 7). Em $17,5 \%$ dos casos a dor foi descrita como intensa (8 a 10). A média de intensidade da dor foi 5,6 e a mediana, 5. A duração da queixa álgica foi prolongada (variando entre 12 dias e 60 meses); a média foi 10 meses e a mediana, 5. Dor há mais de 6 meses foi relatada por $63,2 \%$ dos indivíduos e há mais de 1 ano por $21,1 \%$.

Houve predomínio do sexo masculino $(65,7 \%)$ no grupo 1 e, no grupo 2, a distribuição foi equitativa. Não se observou diferença estatisticamente significante entre a ocorrência de dor, em relação ao sexo.

A faixa etária dominante para ambos os grupos foi a entre 55 e 81 anos (51,5\% no grupo 1 e 43,8\% no grupo 2). Para o grupo 1 , a média de idade foi 52,7 anos e a mediana, 55 anos. Para o grupo 2, a média foi 50,1 e a mediana, 52 anos. Não houve diferença estatisticamente significante na ocorrência de dor, em relação à idade.

$\mathrm{Na}$ maioria dos doentes dos grupos 1 e 2, a escolaridade foi até 4 anos $(54,3 \%$ e $64,9 \%$ respectivamente). A média de escolaridade foi 6,1 anos para os indivíduos do grupo 1 e de 4,9 anos para os do grupo 2. A mediana foi 4 anos para ambos os grupos. Não houve diferença estatisticamente significante na ocorrência de dor, de acordo com a escolaridade.

A maior proporção dos doentes do grupo 1 $(34,3 \%)$ e do' grupo $2(36,9 \%)$ não possuía atividade ocupacional remunerada; estava desempregada ou era composta por donas de casa ou estudantes. Cerca de $1 / 3$ dos individuos estava aposentado (grupo $1=31,4 \%$

e grupo $2=28,0 \%$ ). Não se observou diferença estatisticamente significante entre a ocorrência de dor e o desempenho de atividade ocupacional remunerada.

A média da renda per capita foi 1,8 e, a mediana, 1,5 , para os doentes do grupo 1 e para aqueles do grupo 2 foi 1,4 e, a mediana, 1 . Não se observou diferença estatisticamente significante entre a ocorrência de dor e a renda per capita.

As localizações mais freqüentes do tumor primário nos doentes do grupo 1 foram o sistema digestivo $(20,0 \%)$, o pulmão $(17,1 \%)$ e a mama $(14,3 \%)$. Nos doentes do grupo 2, a localização mais freqüente do tumor primário foi o segmento crânio-cervical $(33,4 \%)$, seguido do sistema digestivo $(17,5 \%)$ e da mama (12,3\%). A ocorrência de tumores de cabeça e pescoço foi pequena no grupo $1(8,6 \%)$ e expressiva no grupo $2(33,4 \%)$. Esta diferença foi estatisticamente significativa, pelo teste de Fisher $(p=0,0003)$.

Quanto à extensão da doença oncológica, observouse que, entre os indivíduos dos grupos 1 e 2, cerca de 40,0\% possuía doença em atividade à distância. Proporção semelhante possuía doença com atividade loco-regional. Não se encontrou diferença estatisticamente significante entre a ocorrência de dor e o tipo de atividade da doença. Os 2 grupos foram semelhantes quanto ao número médio de locais de metástases $(1,3)$. As metástases pulmonares e ósseas foram as mais freqüentes.

Os doentes de ambos os grupos, em sua maioria, conheciam o diagnóstico da neoplasia. Isto ocorreu em cerca de $65,0 \%$ dos indivíduos do grupo sem dor e com dor. Não foi observada diferença estatisticamente significante entre ocorrência de dor e estar ciente do diagnóstico de neoplasia.

\section{Depressão nos doentes dos grupos 1 e 2}

Para se avaliarem aspectos depressivos nos doentes dos grupos 1 e 2 utilizou-se o inventário de depressão de Beck. O escore médio encontrado no grupo 1 foi 10,1 (mediana, 7). Escore superior a 13, foi observado em 10 (28,6\%) doentes. Em 3 (8,6\%), foi superior a 21 pontos.

Nos doentes do grupo 2 (com dor), o escore médio de depressão foi 19,4 (mediana, 19). Escore superior a 13 , foi observado em $38(66,7 \%)$ doentes e igual ou superior a 21, em 25 (43,9\%). Comparando-se os escores obtidos pelos doentes dos grupos 1 e 2 observou-se que a média obtida no grupo $2(19,4)$ foi quase o dobro da obtida no grupo $1(10,1)$. Esta diferença foi estatisticamente significante pela prova de MannWhitney (observado $=4,25$ - crítico $=1,64$ ).

A freqüência da escolha de cada uma das 21 questões do inventário de depressão de Beck estão apresentadas, em número absoluto e em porcentagem, 
no QUADRO 1. Nota-se no grupo 1 (sem dor), que os itens de fatigamento $(57,1 \%)$, preocupações somáticas $(54,3 \%)$ e irritabilidade $(51,4 \%)$ estavam alterados em mais de $50 \%$ dos doentes. Sensação de fracasso, de punição e isolamento social foram referidos por menos de $10 \%$ dos doentes e nenhum informou ter tido ideação suicida. No grupo 2 (com dor), observou-se que aspectos relativos a preocupações somáticas $(91,2 \%)$, fatigamento $(87,7 \%)$, anormalidades do sono $(82,5 \%)$, inibição para o trabalho $(70,1 \%)$, perda de apetite $(68,4 \%)$, humor deprimido $(66,7 \%)$, irritabilidade $(64,9 \%)$, choro $(57,9 \%)$ e perda de satisfação $(50,9 \%)$ estavam alterados em mais de $50 \%$ dos doentes. Nenhum dos 21 aspectos esteve alterado em menos de 10,0\% dos doentes estudados. As alterações depressivas foram em maior número, acometeram maior parcela dos doentes do grupo 2 e apresentaram-se de forma mais intensa que no grupo 1.
A ocorrência de tumores de cabeça e pescoço foi maior no grupo com dor. Para verificar se a diferença nos escores de depressão apresentada pelos grupos estava associada à localização mais freqüente ou à presença de dor, foram aplicados testes estatísticos. As provas de Kruskall Wallis e Mann Whitney revelaram que as diferenças devem-se à presença ou não da dor e não à localização do tumor.

Analisando-se a intensidade da dor e a variável estado depressivo observou-se correlação positiva, estatisticamente significante. Isto é, à dor mais intensa corresponderam escores mais altos no inventário de depressão de Beck. (Prova de Kendall Tau - valor crítico: 1,64 para as correlações positivas observado - 2,73).

QUADRO 1 - Distribuição da frequência de escolha dos itens do inventário de depressão de Beck, pelos doentes do grupo 1 (sem dor) e 2 (com dor). São Paulo, 1994.

\begin{tabular}{lcccc}
\hline ÍTENS & \multicolumn{2}{c}{ GRUPO 1 } & \multicolumn{2}{c}{ GRUPO 2 } \\
& N & \% & N & \% \\
\hline 1- Humor deprimido & 9 & 25,7 & 38 & 66,7 \\
2 - Pessimismo & 13 & 37,1 & 18 & 31,6 \\
3 - Sensação de fracasso & 2 & 5,7 & 12 & 21,1 \\
4 - Perda de satisfação & 10 & 28,6 & 29 & 50,9 \\
5 - Sentimentos de culpa & 9 & 25,7 & 9 & 15,8 \\
6 - Sensação de punição & 3 & 8,6 & 20 & 35,1 \\
7 - Auto-rancor (ódio, aversão) & 7 & 20,0 & 28 & 49,1 \\
8 -Auto-acusação & 12 & 34,3 & 22 & 39,6 \\
9 - Ideação suicida & - & - & 6 & 10,5 \\
10 -Choro & 15 & 42,9 & 33 & 57,9 \\
11- Irritabilidade & 18 & 51,4 & 37 & 64,9 \\
12 - Isolamento social & 2 & 5,7 & 14 & 24,6 \\
13 - Indecisão & 7 & 20,0 & 25 & 43,9 \\
14 - Imagem corporal alterada & 10 & 28,6 & 23 & 40,4 \\
15 - Inibição para o trabalho & 13 & 37,1 & 40 & 70,1 \\
16 - Anormalidades do sono & 16 & 45,7 & 47 & 82,5 \\
17 - Fatigamento & 20 & 57,1 & 50 & 87,7 \\
18 - Perda de apetite & 16 & 45,7 & 39 & 68,4 \\
19 -Perda de peso & 10 & 28,6 & 23 & 40,4 \\
20 - Preocupações somáticas & 19 & 54,3 & 52 & 91,2 \\
21 - Perda da libido & 14 & 40,0 & 25 & 43,9 \\
& & & &
\end{tabular}




\section{DISCUSSÃO}

Dor é sintoma freqüente em doentes com câncer $7,15,17$. No presente estudo, constatou-se freqüência de ocorrência de dor em 62,0\% entre 92 doentes com câncer avançado, atendidos em uma unidade de radioterapia, de um hospital geral. Os dados estão de acordo com os encontrados por alguns autores $8,15,19,31$ e situam-se entre os valores obtidos por outros $1,17,28$

Não foram observadas diferenças estatisticamente significantes nos dados relativos à caracterização pessoal que compreendem sexo, idade, escolaridade, atividade ocupacional remunerada e renda per capita, entre os doentes com e sem dor. As características da afecção neoplásica poderiam ser fatores envolvidos na ocorrência da dor. No entanto, no que tange às características da neoplasia, os grupos apresentaram comportamento semelhante em relação às variáveis: tipo de evolução da doença, número médio de locais de metástases e ciência do diagnóstico de neoplasia. Isto mostra que a presença de dor não foi influenciada por estas variáveis. Quanto aos aspectos de localização do tumor primário, observou-se que a frequência de ocorrência de tumores de cabeça e pescoço foi menor no grupo sem dor que no grupo com dor. Esta diferença foi estatisticamente significante. Relativo à variável depressão, os grupos não se comportaram igualmente. Encontrou-se diferença estatisticamente significante entre $o$ indice de depressão nos doentes com e sem dor. Os doentes com dor apresentaram maiores indices de depressão que os doentes sem dor. A depressão, portanto, associou-se à ocorrência de dor.

Todos os doentes estavam em tratamento ambulatorial e, em sua maioria, submetiam-se a tratamento antineoplásico (cerca de 60\% à quimioterapia e $8 \%$ à radioterapia). Na literatura sobre dor oncológica, é usual utilizar-se a palavra avançado como sinônimo de terminal ${ }^{8}$. Possivelmente, alguns trabalhos analisados no presente estudo, envolveram doentes que, embora a doença tenha sido classificada como em fase avançada, eram, na realidade, doentes terminais, na definição operacional adotada no presente estudo. Isto, talvez, justifique algumas discrepâncias entre os resultados observados no presente estudo com os relatados por outros autores. Ressalta-se a alta ocorrência de dor nos doentes avaliados neste estudo, o que confirma o conceito de que a dor é muito frequente na doença oncológica, principalmente em fases avançadas ${ }^{8}$. Sabe-se que nos países não desenvolvidos a maioria dos tumores é diagnosticada em fase adiantada da doença, onde a cura não é possivel e o controle dos sintomas é o objetivo da assistência. Assim, o alivio da dor é uma das prioridades no atendimento às pessoas com neoplasia.

Cerca de $65,0 \%$ dos doentes de ambos os grupos conhecia o seu diagnóstico de neoplasia maligna. A utilização das palavras câncer ou tumor maligno para descrever sua doença foi o critério para considerar que o doente conhecia o diagnóstico. Observou-se que a variável estar ciente do diagnóstico de neoplasia não interferiu na presença de dor. Este é um resultado muito importante. Em nosso meio, é habitualmente aceito que o fato de ser informado sobre o diagnóstico de uma neoplasia implica em maior sofrimento e induz os doentes a desistirem do tratamento e à ideação suicida. Nesta linha de pensamento, o desconhecimento do diagnóstico de neoplasia protegeria o indivíduo de parte do sofrimento. Sabendo-se que a vivência dolorosa é resultante de fatores biológicos, emocionais e sociais e supondo-se que a ciência do diagnóstico acarreta sofrimento adicional, seria lógico admitir que o doente que sabe estar com neoplasia maligna é mais susceptivel a vivenciar dor, o que, não ocorreu. Na comparação entre os grupos, o fato de o doente conhecer ou não o diagnóstico de câncer não interferiu na ocorrência de dor. Não se encontrou estudo que tivesse analisado esta relação. AHLES et al. ${ }^{2}$ observaram que doentes que julgavam que a presença de dor era sinal de avanço da doença oncológica, experienciaram dores mais intensas do que os que não faziam esta relação.

Aspectos emocionais, entre eles a depressão, também parecem influir na vivência e controle da sintomatologia álgica. Há dúvidas sobre a correlação dor e depressão. Indaga-se se a presença de dor, por si só, pode influir na determinação de depressão ou se, em situações onde a intensidade dolorosa é maior, esta ligação é mais importante. Questiona-se se a depressão no doente oncológico com dor deve-se ao câncer em si ou se a dor tem um papel significativo nesta relação. Diferenças nos métodos diagnósticos de depressão e dor, nas características das populações estudadas e as semelhanças entre os sintomas de dor e depressão, são algumas possiveis causas da grande variação de frequências de ocorrência e concorrem para a existência de dúvidas acerca desta relação. Diversas teorias tentam explicar porque a dor e depressão coexistem. Algumas assumem haver relação de causa e efeito, onde a dor antecede a depressão ou vice-versa. Outras são mais integrativas e não valorizam o fenômeno inicial. Há teorias biológicas e psicossociais 7,36. O modelo cognitivo comportamental admite que, devido à redução das atividades laborativas, sociais e de lazer, que frequentemente acompanha os quadros álgicos, sentimentos de perda de prestigio social, isolamento, perda de atividades prazeirosas e de controle da situação, entre outras, ocasionam depressão. Por outro lado, o indivíduo deprimido, centrado em si mesmo, afastado do convivio social e das atividades laborativas, está mais susceptivel para perceber sensações dolorosas que em outras situações não seriam identificadas, ou o seriam com magnitudes diferentes $7,20,36$ modelo 
neurobiológico de dor e depressão envolve mecanismos bioquímicos similares que resultam na menor disponibilidade de neurotransmissores centrais, principalmente da serotonina e da norepinefrina. Drogas que revertem os quadros depressivos, como os inibidores da monoaminaoxidase (IMAO) atuam bloqueando a ação da enzima responsável pelo metabolismo destas aminas, prolongando sua vida biológica. Os antidepressivos tricíclicos causam inibição da recaptação de serotonina e norepinefrina e ocasionam maior disponibilidade destes neurotransmissores na fenda sinóptica. Estas drogas melhoram não apenas a depressão, como também, a dor ${ }^{12}$. A natureza da associação entre dor e depressão varia entre doentes diferentes e, possivelmente, resulta de um equilíbrio de forças. A associação entre dor e depressão, observada no presente estudo, foi relatada por diversos autores $5,12,23$

Depressão é uma palavra utilizada para descrever um grupo heterogêneo de fenômenos. Humor depressivo, isto é, tristeza, é uma reação normal a frustrações ou perdas. É um dos componentes da depressão. A depressão é constituída por vários sinais e sintomas. Há diminuição acentuada no interesse e no prazer pelas atividades anteriormente prazeirosas, perda ou aumento significativo de peso, insônia ou hipersônia, agitação ou retardo psicomotor, fadiga ou perda de energia, sentimentos excessivos ou inapropriados de culpa, diminuição na velocidade do pensamento e da concentração e pensamentos de morte e suicídio recorrentes, frente a situações em que não foi possivel o estabelecimento de causas orgânicas e não ocorreu perda de entes queridos. A depressão compromete, de maneira significativa, o relacionamento familiar, o desempenho profissional e o convivio social. Sabe-se que sua ocorrência piora o prognóstico de outras doenças e contribui para a ocorrência de outras queixas como dor e fadiga, entre outras ${ }^{12}$. A consequência mais grave da depressão não tratada é o suicídio. O quadro patológico em que episódios de depressão se alternam com periodos de mania é denominado depressão bipolar ${ }^{30}$. A caracterização do tipo de depressão apresentada pelos doentes não foi objetivo deste trabalho.

O inventário de depressão de Beck, utilizado no presente estudo, é um instrumento de autoavaliação. Escalas de auto-avaliação podem ser vulneráveis pela possibilidade de o doente, deliberadamente, negar ou exarcebar sintomas, enfatizar traços socialmente mais aceitos ou responder nos extremos da gama de respostas. Além disso, o nível cultural, a posição social e étnica podem interferir na compreensão e significado atribuído aos termos que o compõem. Podem também ser inadequadas para pacientes intensamente deprimidos, visto a limitação e ausência de motivação para respondê-las. Apresentam, como vantagens, a eliminação das diferenças na interpretação dada pelos avaliadores e o fato de serem instrumentos práticos e não necessitarem de profissional especializado para aplicá-los. Além disso, a auto-avaliação e a percepção dos médicos sobre sintomas depressivos se correlacionam ${ }^{21}$ e a auto-avaliação é fundamental para a compreensão de sintomas subjetivos". Convém ressaltar que a auto-avaliação são medidas de rastreamento e não diagnósticas 36

Os indivíduos dos grupos com e sem dor apresentaram comportamento semelhante em relação a todas as variáveis estudadas, excetuando-se a variável estado depressivo. Ocorreu diferença estatisticamente significante entre os escores de depressão apresentados pelos doentes sem dor (média 10,1) e com dor (média 19,4). Isto é, a depressão foi um fator que se associou à ocorrência de dor. Ainda, quando se utilizou 14 como ponto de corte para identificar depressão $28,6 \%$ dos doentes sem dor foi considerado deprimido. No grupo com dor, este número elevouse para $66,7 \%$. Utilizando-se 21 como ponto de corte, encontrou-se, no grupo sem dor, apenas $8,6 \%$ dos individuos classificados como deprimidos e, no grupo com dor, este percentual manteve-se elevado: $43,9 \%$. Conclui-se que houve maior ocorrência de depressão nos doentes com dor.

Entre os doentes com dor, as alterações depressivas foram em maior número e acometeram maior número de doentes. Nos doentes sem dor, 3 aspectos estavam alterados em mais de $50 \%$ dos avaliados: fatigamento, preocupações somáticas e irritabilidade. Entre os doentes com dor, 9 aspectos encontravam-se alterados em mais da metade dos doentes: preocupações somáticas, fatigamento, anormalidades do sono, inibição para o trabalho, perda de apetite, humor depressivo, irritabilidade, choro e perda da satisfação. Observou-se também que, mesmo os sinais e sintomas somáticos, que são comuns à sindrome depressiva e ao câncer, como alterações do sono, anorexia, perda de peso, fatigamento e preocupações somáticas apresentaram-se muito mais frequentes no grupo com dor que nos doentes sem dor. Estes dados estão em acordo com os observados por CHATUVERDI; MAGUIRE ${ }_{12}$

CLARK et al.13 desenvolveram estudo para discriminar quais itens do Inventário de Depressão de Beck poderiam melhor prever a intensidade da depressão. Concluíram que a presença concomitante de ideação suicida, sensação de fracasso, sensação de culpa, isolamento social, indecisão e perda de satisfação pode ser tida como critério para a determinação da intensidade da depressão e não é mascarada pela presença de doença física. Os dados observados na presente pesquisa, mostram que a sensação de fracasso, o isolamento social, a indecisão e a perda 
da satisfação foram 2 a 3 vezes mais frequentes nos doentes com dor do que nos doentes sem dor. Ideação suicida, que não ocorreu nos doentes sem dor, foi referida por $6(10,5 \%)$ dos doentes com dor. Dor incontrolada é o maior fator para o suicídio relacionado ao câncer ${ }^{9}$. A ideação suicida é frequente, principalmente na doença avançada, e parece representar, para o doente, uma opção para o controle da situação ${ }^{9}$. Em estudo sobre as características de doentes terminais, que envolveu 90 indivíduos, observou-se que 20\% verbalizou ideação suicida. Destes, 2 doentes suicidaram-se ${ }^{16}$. Alguns doentes do presente estudo poderiam ser considerados como de risco para suicídio. Tomou-se o cuidado de, após a entrevista, avisar aos doentes identificados como deprimidos, que o serviço possuía atendimento psicológico para os quais poderiam ser encaminhados. Também os doentes que estavam com dor muito intensa foram avisados da existência do Ambulatório de Dor na instituição, ao qual poderiam recorrer.

Foi ainda observada a existência de correlação positiva, estatisticamente significativa, entre a intensidade da dor e os escores de depressão mais elevados. Este é um dado pouco explorado na literatura. BUCKERG et al. 10 observaram que a dor era mais intensa nos doentes oncológicos mais deprimidos, mas esta relação não foi estatisticamente significativa. SPIEGEL; BLOOM 31 estudaram a relação entre dor (intensidade, duração e significado simbólico) e variáveis médicas (prescrição de analgésicos) e psicológicas (anormalidades do humor, respostas de enfrentamento da doença e a percepção do suporte familiar). Obtiveram correlação positiva, estatisticamente significante, entre a intensidade da dor e anormalidades do humor, a crença do doente de que a dor indicava piora da doença e maior uso de analgésicos. LEOPOLD et al 23 também observaram relação positiva entre a presença de depressão e a intensidade da dor.

Dor e depressão caminham juntas e uma agrava a outra. Observou-se, nesta pesquisa, que os escores de depressão eram significantemente mais elevados no grupo de doentes com dor, comparados aos sem dor, e que à dor de intensidade maior correspondia escores mais altos de depressão. Como os aspectos biológicos, emocionais, culturais, entre outros, participam da interpretação do fenômeno doloroso, é de importância máxima avaliar e tratar cada um deles, quando o objetivo é o controle da dor.

\section{CONCLUSÕES}

A busca de possiveis relações entre a presença de dor e diversas variáveis mostrou que a ocorrência de dor não se associou às variáveis: sexo, idade, escolaridade, renda per capita, atividade ocupacional remunerada, atividade da doença, número de locais de metástases e ciência, pelo doente, do diagnóstico de doença oncológica. Constatou-se associação entre dor e estado depressivo. Os doentes com dor apresentaram, no Inventário de Depressão de Beck, escores depressivos significativamente mais elevados que os doentes sem dor. Tumores de cabeça e pescoço foram mais frequentes nos doentes do grupo com dor. A análise estatística mostrou que os escores de depressão não diferiram em relação à localização do tumor, mas diferiram, significativamente, segundo a presença ou ausência de dor.

Constatou-se correlação positiva entre o escore de depressão e a intensidade da dor. Mais altos escores de depressão correlacionaram-se à dor de maior intensidade.

A importância clínica desses achados é inquestionável. Todos os profissionais que atuam junto a doentes com dor crônica em geral e, em especial, junto a doentes oncológicos, devem estar alertas para a identificação da presença de depressão. Dor e depressão frequentemente coexistem e uma agrava a outra. Doentes com dor e deprimidos apresentam prejuízos para a vida diária de magnitude superior àqueles que, embora com dor, não apresentam depressão. A identificação precoce e o tratamento adequado desses agravos contribui para a melhora da qualidade de vida dos doentes.

\section{REFERÊNCIAS BIBLIOGRÁFICAS}

1. AHLES, T.A.; RUCKDESCHEL, J.C.; BLANCHARD, E.B. Cancer-related pain I: prevalence in an out patient setting as a function of stage of disease and type of cancer. $\boldsymbol{J}$. Psychosom. Res., v.28, n.2, p.115-9, 1984.

2. AHLES, T.A. et al. The multidimension nature of cancer-related pain. Pain, v.17, n.3, p.277-88, 1983.

3. BECK, A.T. Terapia cognitiva da depressão. Rio de Janeiro, Zahar, 1982. p.385-7: Apêndice:- materiais.

4. BECK, A.T. et al. An inventory for measuring depression. Arch. Gen. Psychiatry, v.4, p.561-71, 1961.

5. BERNABEI R. et al. Management of pain in elderly patients with cancer. JAMA, v.279, n. 23, p.1914-5, 1998.

6. BERQUÓ, E.S.; SOUZA, J.M.; GOTLIEB, S.L.D. Bioestatistica. São Paulo, EPU, 1981. cap. 10, p.193-201: Teste de hipóteses.

7. BOND, M.R. Cancer pain: psychological substrates and therapy. In: FIELDS, H.L.; DUBNER R. CERVERO, F. Advances in pain research and therapy: proceeding of the Fourth World Congress on Pain. New York, Raven, 1985. v.9. p.559-67.

8. BONICA, J.J. Treatment of cancer pain: current status and future needs. In: FIELDS, H.L.; DUBNER R.; CERVERO, F. Advances in pain research and therapy: proceeding of the Fourth World Congress on Pain, New York, Raven, 1985. v.9, p.589-615. 
9. BREITBART, W. Psychiatric management of cancer pain. Cancer, v.63, p.2336-42, 1989. suppl.

10. BUCKERG, J.; PENMAN, D.; HOLLAND, J.C. Depression in hospitalized cancer patients. Psychosom. Med., v.46, n.3, p.199-212, 1984

11.CASSEM, E.H.; Depression and anxiety secondary to medical illness. Psychiatr.Clin.North Am. v. 13, n. 4, p.597-611, 1990.

12. CHATUVERDI S.K.; MAGUIRE, G.P. Persistent somatization in cancer: a controlled follow-up study. J.Psychosom Res.

V. 45, n.3, p. 249-56, 1998.

13. CLARK, D.C.; CAVANAUGH, S.A; GIBBONS, R.D. The core symptoms of depression in medical and psychiatric patients. J. Nerv. Ment. Dis., v.171, n.12, p.705-13, 1983.

14. CLEELAND, C.S. Research in cancer pain- what we know and what we need to know. Cancer, 67, n.3,. p.823-7, 1991. suppl.

15. CLEELAND, C.S. et al. Pain and its treatment in outpatients with metastatic cancer, N. Engl. J. Med., v.330, n.9, p.5926, 1994.

16. COYLE, N. Characteristics of advanced cancer as terminall illness. J.Pain Symptom..Manage., v.5, n.2, p.83-93, 1990.

17. DAUT, R.L.; CLEELAND, C.S. The prevalence and severity of cancer pain. Cancer, v.50, n.1, p.1913-8, 1982.

18. DEL PORTO, J.A. Aspectos gerais das escalas para avaliação de depressão. In: CENTRO DE PESQUISA EM PSICOBIOLOGIA CLÍNICA. São Paulo, 1989. p.93-105.

19. GREENWALD, H.P. et al. The prevalence of pain in four cancers. Cancer, v.60, n.10, p.2563-9, 1987.

20. HERR, K.A; MOBILY, P.R. Chronic pain and depression. J. Psych.Nurs., v.30, n.9, p.7-12, 1992.

21. HIRAGA, $K$. et al. The incidence of cancer pain and improvement of pain management in Japan, Postgrad. Med. J., v.67, p.14-25, 1991. Supp 1.2

22. HUSKISSON, E.C. Measurement of pain. Lancet, v.2, n.7889, p.1127-31, 1974.

23.
LEOPOLD, K.A. et al. Prevalence of mood disorders and utility of PRIME-MD in patients undergoing radiation therapy. Int $J$ Radiat Oncol.Biol.Phys. v.42, n. 5, p. 1105-12, 1998.

24. MERSKEY, H. Pain and psychological medicine. In: WALL PD, MELZACK R. Textbook of pain. 3rd. ed. Edinburgh, Churchill Livingstone, 1994. cap.47, p.903-20.

\section{ORGANIZACION MUNDIAL DE LA SALUD Alivio del} dolor en el cancer. Ginebra, 1987.

26. PAREDES, H. et al. Tratamiento farmacologico en tres pasos del dolor en cancer: experiencia preleiminar en Chile, Rev. Med. Chil., v.116, n.5, p.433-8, 1988.

27. PASSIK, S.D. et al. Oncologist's recognition of depression in their patient's with cancer. J Clin.Oncol. v.16, n.4, p. 1594-600, 1998.

28. PORTENOY, R.K. et al. Pain in ambulatory patients with lung or colon cancer: prevalence, characteristics, and effects, Cancer, v.70, n.6, p.1616-24, 1992.

29. SIEGEL, S. Estatística não paramétrica. São Paulo, McGraw-Hill, 1975.

30. SOCIEDADE BRASILEIRA DE PSIQUIATRIA CLÍNICA. Depressão em atenção primária:detecção, diagnóstico e tratamento. Ribeirão Preto, 1994.

31. SPIEGEL, D.; BLOOM, J.R. Pain in metastatic breast cancer. Cancer, v. 52, n.2, p.341-5, 1983.

32. STJERNSWARD, J. Cancer pain relief: an important global public health issue. In: FIELDS, H.L.; DUBNER R. CERVERO, F. Advances in pain research and therapy: proceedings of the Fourth World Congress on Pain, 1985, v.9, p.555-7.

33. TEIXEIRA, M.J. Tratamento neurocirúrgico da dor. In: RAIA, A.A.; ZERBINI, E.J. Clínica cirúrgica Alipio Correa Neto. 4ed. São Paulo, Sarvier, 1988. v.2. cáp.62, p.541-72.

34. VAINIO, A. Practising physician's experiences of treating with cancer pain. Acta Oncol., v.28, n.2, p.177-82, 1989

35. VENTAFRIDA, V. et al. A validation study of the who method for cancer pain relief. Cancer, v.59, n.4, p.850-6, 1987.

36. WARD, N.G. Pain and depression In: BONICA, J.J. The management of pain. 2.ed. Philadelphia Lea \& Febeger, 1990, cap. 18 , p.310-19. 